\title{
repisälud
}

This is the peer reviewed version of the following article:

Bueno, H., Rossello, X., Pocock, S. J., Van de Werf, F., Chin, C. T., Danchin, N., . .. Huo, Y. (2019). In-Hospital Coronary Revascularization Rates and PostDischarge Mortality Risk in Non-ST-Segment Elevation Acute Coronary Syndrome. Journal of the American College of Cardiology, 74(11), 1454-1461. doi:10.1016/j.jacc.2019.06.068

which has been published in final form at: https://doi.org/10.1016/j.jacc.2019.06.068 


\section{In-hospital Coronary Revascularization Rates and Post-discharge Mortality Risk in Non-ST-segment Elevation Acute Coronary Syndrome}

Brief title: Revascularization and post-discharge mortality in ACS

Héctor Bueno, MD, PhD, ${ }^{\mathrm{a}, \mathrm{b}, \mathrm{c}}$ Xavier Rossello, MD, PhD, ${ }^{\mathrm{a}, \mathrm{d}, \mathrm{e}}$ Stuart Pocock, PhD, ${ }^{\mathrm{d}}$ Frans Van de Werf, MD, PhD, ${ }^{\mathrm{f}}$ Chee Tang Chin, MD, ${ }^{\mathrm{g}}$ Nicolas Danchin, MD, PhD, ${ }^{\mathrm{h}}$ Stephen W-L Lee, MD, $\mathrm{PhD},{ }^{\mathrm{i}}$ Jesús Medina, $\mathrm{PhD},{ }^{\mathrm{j}}$ Yong Huo, $\mathrm{MD}, \mathrm{PhD}^{\mathrm{k}}$

${ }^{a}$ Centro Nacional de Investigaciones Cardiovasculares (CNIC), Madrid, Spain; ${ }^{b}$ Instituto de investigación $i+12$ and Cardiology Department, Hospital Universitario 12 de Octubre, ${ }^{c}$ Facultad de Medicina, Universidad Complutense de Madrid, Madrid, Spain; ${ }^{d}$ London School of Hygiene and Tropical Medicine, London, UK; ${ }^{e}$ CIBER de enfermedades CardioVasculares, Madrid, Spain; ${ }^{f}$ Department of Cardiovascular Sciences, University of Leuven, Leuven, Belgium; ${ }^{g}$ National Heart Centre Singapore, Singapore; ${ }^{h}$ Hôpital Européen Georges Pompidou \& René Descartes University, Paris, France; ${ }^{i}$ Queen Mary Hospital, Hong Kong SAR, China $;{ }^{j}$ Global Medical Affairs, AstraZeneca, Madrid, Spain; ${ }^{k}$ Beijing University First Hospital, Beijing, China.

Funding: The EPICOR and EPICOR Asia studies were funded by AstraZeneca.

Disclosures: H Bueno receives research funding from the Instituto de Salud Carlos III, Spain (PIE16/00021 \& PI17/01799), AstraZeneca, BMS, Janssen and Novartis, has received consulting fees from AstraZeneca, Bayer, BMS-Pfizer, Novartis, and speaking fees or support for attending scientific meetings from AstraZeneca, Bayer, BMS-Pfizer, Ferrer, Novartis, and MEDSCAPEthe heart.org. X Rossello has nothing to disclose. S Pocock has received research funding from AstraZeneca. F Van de Werf has received consulting fees and research grants from Boehringer Ingelheim, Merck and Sanofi, and consulting and speaking fees from Boehringer Ingelheim, Roche, Sanofi, AstraZeneca, and The Medicines Company. CT Chin has received consulting or speaking fees from AstraZeneca, Merck, Servier, and Medtronic. N Danchin has received consulting or speaking fees from Amgen, AstraZeneca, Bayer, BMS, Boehringer-Ingelheim, MSD, NovoNordisk, Pfizer, Sanofi-Aventis, and Servier. SW-L Lee has nothing to disclose. J Medina is an employee of AstraZeneca. Y Huo has nothing to disclose.

Acknowledgements: Editorial assistance was provided by Carl V Felton $\mathrm{PhD}$, Paragon (Knutsford, Cheshire, UK), funded by AstraZeneca. CNIC is supported by the Spanish Ministry of Economy and Competitiveness (MINECO) and the Pro-CNIC Foundation and is a Severo Ochoa Center of Excellence (MINECO award SEV-2015-0505). X.R. has received support from the SEC-CNIC CARDIOJOVEN fellowship program.

\section{Address for Correspondence:}

Héctor Bueno, MD, PhD

Centro Nacional de Investigaciones Cardiovasculares (CNIC)

Melchor Fernandez Almagro, 3

28029-Madrid, Spain

Tel: +34 914531200

E-mail: hector.bueno@cnic.es 
Tweet: @RosselloXavier: "Better 2-year survival in hospitals, countries and regions with higher revascularization rates after ACS: Revascularization or higher quality care?" 


\begin{abstract}
Background: The relationship between in-hospital coronary revascularization rate (CRR) and post-discharge mortality rates in survivors of non-ST-segment elevation acute coronary syndrome (NSTE-ACS) at a system level is unclear.

Objectives: To evaluate CRR and 2-year post-discharge mortality rate (2YMR) in NSTE-ACS. Methods: CRR and 2YMR were analyzed by hospital rate of CRR (in deciles), by country and by world region in 11,931 patients with NSTE-ACS who survived to discharge and were enrolled in the EPICOR and EPICOR-Asia registries, twin multinational, observational, prospective cohort studies.

Results: Significant differences in patient baseline characteristics, medical therapies, CRR and 2YMR were found. Mean CRR ranged from $0.0 \%-96.8 \%$ in the first and tenth decile, respectively ( $\mathrm{p}<0.001$ ), from $12.3 \%$ in Romania to $92.4 \%$ in Slovenia $(\mathrm{p}<0.001)$, and from $53.9 \%$ in South East Asia (SEAsia) to $90.4 \%$ in South Korea-Singapore-Hong Kong. 2YMR varied significantly between hospital deciles of CRR (3.6\% in tenth decile vs $9.2 \%$ in first decile; $\mathrm{p}<0.001$ ), countries (lowest 1.5\% in Slovenia; highest 19.4\% in Malaysia, $\mathrm{p}<0.001$ ), and regions (lowest South Korea-Singapore-Hong Kong, 3.8\%; highest SEAsia, 11.7\%; p<0.001). Poisson regression models, adjusted for 15 mortality predictors showed a significant inverse association between CRR and 2YMR for hospitals $(\mathrm{r}=-0.90$, $\mathrm{p}<0.001)$, countries $(\mathrm{r}=-0.65, \mathrm{p}<0.001)$, regions $(\mathrm{r}=-0.87, \mathrm{p}=0.005)$.

Conclusions: Higher CRRs at hospital, country and world region level are strongly associated with higher post-discharge survival, suggesting CRR as a marker of higher system quality.
\end{abstract}




\section{Condensed Abstract}

The relationship between in-hospital coronary revascularization rates (CRR) and 2-year postdischarge mortality rates (2YMR) was evaluated at a system level in 11,931 NSTE-ACS hospital survivors enrolled in the EPICOR and EPICOR-Asia registries. CRR and 2YMR varied between hospitals, countries and regions. Multivariable Poisson regression models adjusted for 15 mortality predictors showed a significant inverse association between CRR and 2YMR at a hospital level $(\mathrm{r}=-0.90, \mathrm{p}<0.001)$, country level $(\mathrm{r}=-0.65, \mathrm{p}<0.001)$, and region level $(\mathrm{r}=-$ $0.87, \mathrm{p}=0.005)$. These findings suggest that $\mathrm{CRR}$ may be a marker of higher system quality.

Keywords: Acute coronary syndrome; coronary revascularization; mortality; risk, country, region 

Abbreviations and Acronyms
2YMR = 2-year post-discharge mortality rate
$\mathrm{CABG}=$ coronary artery bypass grafting
$\mathrm{CI}=$ confidence interval
$\mathrm{CRR}=$ coronary revascularization rate
EPICOR = long-tErm follow uP of antithrombotic management patterns In acute CORonary syndrome patients
NSTE-ACS = non-ST-segment elevation acute coronary syndrome
NSTEMI = non-ST-segment elevation myocardial infarction
$\mathrm{PCI}=$ percutaneous coronary intervention
$\mathrm{SD}=$ standard deviation
STEMI = ST-segment elevation myocardial infarction 


\section{Introduction}

Patients with non-ST-segment elevation acute coronary syndrome (NSTE-ACS) are, in general, at high risk of recurrent coronary events and mortality (1). Guidelines recommend an early invasive strategy - that is, coronary angiography and coronary revascularization when feasible - for a majority of these patients, in particular those at high or intermediate risk $(2,3)$. Although there has been progress in recent years (4), there is still significant variation between hospitals, countries and regions in coronary revascularization rate $(\mathrm{CRR})$ for these patients $(1,5)$. Further, the long-term prognostic implications of differences in clinical practice regarding early coronary revascularization for NSTE-ACS patients are largely unknown. The present work was, therefore, designed to assess whether the rate of coronary revascularization performed during index admission in patients with NSTE-ACS, measured at a healthcare system level (hospital, country, region) is independently associated with post-discharge survival. This represents a posthoc analysis of the long-tErm follow uP of antithrombotic management patterns In acute CORonary syndrome patients (EPICOR) and EPICOR Asia studies. 


\section{Methods}

\section{Patients}

All patients with a final diagnosis of NSTE-ACS enrolled in 2 twin prospective studies: EPICOR (NCT01171404, 5,625 patients from 555 hospitals in 20 countries across Europe and Latin America between September 2010 and March 2011), and EPICOR Asia (NCT01361386, 6,306 patients from 219 hospitals from 8 Asian countries between June 2011 and May 2012) $(6,7)$, were considered.

\section{Site Selection}

National coordinators in each country were responsible for site selection according to the national proportion of patients treated at hospitals with and without invasive cardiac intervention facilities, with the intention of achieving a fair representation of real-life practice at a country level.

\section{Definitions}

NSTE-ACS comprised non-ST-segment elevation myocardial infarction (NSTEMI) (chest pain/discomfort, lack of persistent ST-segment elevation, left bundle branch block or intraventricular conduction disturbances, and elevation of cardiac biomarkers [creatine kinaseisoenzyme MB or troponins] with at least 1 value above the 99th percentile of the upper reference limit) and unstable angina (clinical presentation consistent with rest angina or on minimal exercise, with transient ST-T changes in the electrocardiogram and no significant increase in biomarkers of necrosis, but objective evidence of ischemia by non-invasive imaging, or significant coronary stenosis on angiography). Coronary revascularization was defined as the performance of any kind of percutaneous coronary intervention (PCI) or coronary artery bypass grafting (CABG) performed during index hospitalization for NSTE-ACS (6). 


\section{Data Collection}

Patient characteristics, pre-hospital care, hospital features, in-hospital care, including medical therapy, invasive and noninvasive interventions, coronary angiography results and coronary revascularization, were collected. Follow-up was prospectively performed through periodically scheduled, structured telephone calls assessing medical therapy continuation, the occurrence of cardiovascular events, and mortality up to 2 years after discharge (6).

\section{System-level Analysis}

The relationship between CRR during index hospitalization and 2-year post-discharge all-cause mortality rate (2YMR) was assessed at a system level using 3 different approaches: individual hospital level, country level and region level. The 743 participating hospitals that recruited NSTE-ACS patients were divided according to the decile of in-hospital CRR, and these were correlated with 2YMRs. Across the 28 participating countries, the national CRR were correlated with the national 2YMRs. Finally, 8 geographical regions were defined: Southern Europe (France, Greece, Italy and Spain), Northern Europe (Belgium, Denmark, Finland, Germany, Luxembourg, the Netherlands, Norway, and the United Kingdom [UK]), Eastern Europe (Poland, Romania, Slovenia, and Turkey), Latin America (Argentina, Brazil, Mexico, and Venezuela), China, India, South East Asia (Malaysia, Vietnam and Thailand) and South Korea-Singapore-Hong Kong (1,8), and regional CRR were correlated with average regional 2YMRs.

\section{Statistical Analysis}

Continuous parameters are mostly presented as mean (standard deviation [SD]) and groups. Categorical data are expressed as percentages and compared across deciles, countries and regions using Chi-square tests. 
Poisson regression models were used to estimate $2 \mathrm{YMR}$ according to the revascularization status, adjusted for 15 known predictors of 2-year mortality in the overall study population. These predictors were previously obtained from a risk scoring system using Cox regression to estimate 2YMR in individual patients with acute coronary syndrome and with well reported goodness-of-fit, discriminatory power and internal validation (9). Predictors, ranked by predictive strength, were: age, low left ventricular ejection fraction, elevated serum creatinine at admission, poor EuroQol-5 Dimensions score, low hemoglobin at admission, previous cardiac disease, previous chronic obstructive pulmonary disease, elevated blood glucose at admission, prescription of diuretics at discharge, male sex, lower educational level, prescription of an aldosterone inhibitor at discharge, low body mass index, in-hospital cardiac complications, and Killip class. After obtaining the patient-level adjusted 2YMR, the data were collapsed to obtain the mean adjusted 2YMR for each level of analysis (i.e., by hospital deciles, countries and regions). We then plotted them against their corresponding percentage of revascularization, and estimated the Pearson's correlation coefficient, weighted by group size, to determine the strength of the association between 2YMR and CRR. All probability values were 2-sided and $p$ values of $<0.05$ were considered as statistically significant. All statistical analyses were performed using STATA software, version 13.1 (Stata Corp, College Station, TX, USA). 


\section{Results}

The study cohort comprised 11,931 patients recruited in 743 hospitals from 28 countries of 8 world regions. The mean age was 62.8 years (SD, 11.6), 70.5\% male, with high prevalence of risk factors: 7,530 (63.7\%) hypertensive, 4,746 (42.5\%) currently smoking, 4,242 (37\%) with hypercholesterolemia, 3,234 (27.4\%) with diabetes mellitus; and high prevalence of prior cardiovascular disease $(5,039,43 \%)$. A majority of patients were admitted in Killip class I $(6,173,82.9 \%), 3,602(31.9 \%)$ presented with hemoglobin $<13 \mathrm{mg} / \mathrm{dl}, 2,815(24.6 \%)$ with creatinine $\geq 1.2 \mathrm{mg} / \mathrm{dl}$, and 2,135 (20.3\%) with blood glucose $>160 \mathrm{mg} / \mathrm{dl}$. There were substantial differences in patient baseline profiles and medical management across hospital deciles of CRR (Table 1 and Supplementary Table 1), countries (data not shown), and regions (Supplementary Table 2). The in-hospital CRRs were 0\% in hospitals belonging to the first decile, $56.8 \%$ for hospitals in the fifth decile and $96.8 \%$ in hospitals of the tenth decile (Table 1). Compared with other hospitals, those from the lowest decile of CRR were less frequently tertiary/university centers $(26 \%)$, and less often had catheterization laboratories $(31 \%)$ or participated in a $24 / 7$ primary PCI network $(70 \%)$.

CRRs also differed among countries, ranging from $12.3 \%$ in Romania to $92.4 \%$ in Slovenia, $\mathrm{p}<0.001$; Supplemental Table 3), and regions, ranging from 53.9\% in South East Asia to $90.4 \%$ in South Korea-Singapore-Hong Kong ( $<$ 0.001). Complete 2-year follow-up was achieved in 10,275 patients $(86.1 \%)$. The mean follow-up time for the rest of patients $(\mathrm{n}=1,656,13.9 \%)$ was $385 \pm 222$ days. Two-year post-discharge mortality rates also varied significantly between hospital deciles $(3.6 \%$ for decile $10,9.2 \%$ for decile $1 ; \mathrm{p}<0.001)$ countries (lowest $1.5 \%$ in Slovenia, highest $19.4 \%$ in Malaysia, $\mathrm{p}<0.001$ ), and regions (lowest $3.8 \%$ in South Korea-Singapore-Hong Kong, highest, $11.7 \%$ in South East Asia, p < 0.001). 
Using Poisson regression models that adjust for the 15 previously identified independent predictors of post-discharge mortality (Figure 1) (9), we showed a significant difference in the estimated 2YMR between revascularized and non-revascularized patients: 5.2\% (95\% confidence interval [CI] 4.5-5.8\%) versus 9.5\% (95\% CI 8.5-10.4\%), respectively (p < 0.001). Moreover, a significant correlation exists between average in-hospital CRR and adjusted 2YMR at hospital level $(\mathrm{r}=-0.90, \mathrm{p}<0.001)$, country level $(\mathrm{r}=-0.65, \mathrm{p}<0.001)$ and region level $(\mathrm{r}=$ $-0.87, \mathrm{p}=0.005)$ (Central illustration). 


\section{Discussion}

This large, prospective, international cohort study of patients who were discharged alive from hospital after NSTE-ACS shows that the rate of coronary revascularization performed during index hospitalization is inversely associated with 2-year covariate-adjusted mortality rates measured at a hospital, national or regional level. Our findings align well with and support guideline recommendations for coronary revascularization if significant coronary artery disease is present in patients presenting with NSTE-ACS $(2,3)$. They also support the suggestion of considering early invasive strategy as a hospital quality indicator for patients with NSTEMI (10).

We, and others, have previously shown that there are marked regional differences in the management and prognosis of patients with ST-segment elevation myocardial infarction (STEMI) (1) and NSTE-ACS (5,8,11-13), which cannot be explained by baseline patients characteristics alone. In this study, we show that important differences in revascularization rates exist not only at a regional but at a country and hospital level, and that these differences are associated with long-term survival at any level of analysis. This consistent association is relevant as it suggests that patients admitted to facilities offering early coronary revascularization have better long-term outcomes. However, this does not necessarily mean that the mortality benefit is explained only by coronary revascularization itself. Other aspects of care associated with providing early intensive care in these hospitals may also play a role.

A number of other studies, mostly observational, support the hypothesis of a survival benefit associated with coronary revascularization in the early phase of NSTE-ACS. A recent study showed a significant risk-adjusted reduction in 6-month mortality in the UK during the last decade, following a marked increase in the use of coronary angiography and revascularization (4). We, and others, had previously described that NSTE-ACS patients undergoing an invasive 
strategy and coronary revascularization, either PCI or CABG, showed improved survival compared with medically treated patients (14-16). A recent analysis of the Atherosclerosis Risk in Communities (ARIC) surveillance study showed that early PCI (under $24 \mathrm{~h}$ after symptom onset) in hospitalized NSTEMI patients was associated with improved 28-day survival, especially in high-risk patients, but not at 1 year (17). Although using a wider time frame (1 month) for revascularization, a large Canadian cohort study assessing the relationship between coronary revascularization and 1-year prognosis showed consistent results, with a lower risk of death or cardiovascular hospitalization among those undergoing revascularization (18).

There are, however, alternative explanations to a direct beneficial effect of coronary revascularization. The first is selection bias. It is known that patients undergoing an invasive strategy and receiving coronary revascularization are usually younger, show less comorbidity and are at lower risk than medically managed patients $(14,19,20)$. Although this is true, the comprehensive adjustment of our model and the consistency of the effect found at any level of CRR (i.e., hospital deciles) suggest that the effect cannot fully be explained by differences in baseline characteristics. One study comparing the differences in management and outcomes of acute myocardial infarction between all UK and Swedish hospitals reported that coronary revascularization in patients with NSTEMI was associated with a lower 1-year mortality risk difference between hospitals in the highest and lowest revascularization quarter when adjusted for patient case mix $(9 \%$; $95 \%$ CI, $-1 \%$ to $14 \%$, in Sweden, and $14 \%$; $95 \%$ CI, $8 \%$ to $20 \%$, in the UK) (21). A second alternative explanation is that the differences observed in revascularization rates may be a reflection of differences in the quality of care seen from a wider perspective provided by the different healthcare systems or hospitals. In fact, our study shows significant differences in the use of dual antiplatelet therapy, beta-blockers or statins across hospitals from 
different deciles of CRR, as well as between countries and regions. The use of these treatments is a quality indicator for the management of myocardial infarction (10), which has been validated as a prognostic factor as well $(10,22)$. However, although there is some degree of association between quality indicators for the management of myocardial infarction, this association is, at best, modest (22).

At the hospital level, despite the important differences between hospitals within different deciles of CRR (i.e. those in the first decile less frequently tertiary centers, less often with catheterization laboratories or participating in STEMI networks), we cannot say that hospitals with the lowest CRRS were either spoke or hub hospitals as there was a large variation in their characteristics, ranging from hospitals without catheterization laboratory to hospitals with catheterization laboratory and 24/7 PCI programs. In fact, although Northern Europe participated with the lowest proportion of hospitals with on-site catheterization laboratory, they had the highest levels of CRR, indicating a highly efficient referral network. On the contrary, a number of countries, mostly from Asia, participated only with hospitals with catheterization laboratory facility but showed much lower rates of coronary angiography or revascularization (8). The fact that the hospitals with zero CRRs were the ones enrolling the lowest number of patients (mean 8 patients, $53 \%$ recruited $\leq 5$ patients) may explain in part this finding.

\section{Limitations}

A number of limitations should be considered in this study: 1) It is important to highlight that a causal relationship between initial coronary revascularization and post-discharge mortality risk cannot be concluded from these findings. Despite the thorough risk adjustment, its proven value as a quality indicator, and the reasoning as a wider marker of system quality, the causal 
effect of coronary revascularization remains hypothetical. 2) It is not possible to rule out the possibility that differences in post-discharge mortality may be explained in part by differences in hospital mortality (i.e., initial survival of higher risk patients may translate into higher post-discharge mortality). Also, mortality related to elective post-discharge revascularization procedures is not considered, which may be particularly relevant for CABG. However, interestingly, the regions with highest baseline risk did not show the highest post-discharge mortality rate. 3) The lack of information on the cause of death precludes the inference of a potential mechanism for a survival advantage. 4) While both registries were designed to recruit representative patients from representative centers in each country, we cannot rule out a bias in regard to the type of hospitals recruiting patients. Hence, some caution is needed to extrapolate our findings regarding geographic differences, since these centers might not be fully representative of the whole country. 5) Despite its wide representation, this is not a global study as important regions are not included. However, it is likely that similar findings would have been found had more regions been included in the study.

In conclusion, higher average rates of coronary revascularization performed during index hospitalization in patients presenting with NSTE-ACS, measured at a hospital, country and world region level, are independently associated with lower adjusted 2-year mortality rates. 


\section{PERSPECTIVES}

Competency in medical knowledge: Despite international guidelines recommendations, there is huge variability in the rates of in-hospital coronary revascularization rates among hospitals, countries and world regions. Higher coronary revascularization rates are inversely correlated with lower adjusted post-discharge mortality risks at all levels of analysis.

Competency in patient care: Interventions to ensure that a majority of NSTE-ACS patients undergo coronary angiography and, if needed, coronary revascularization during index hospitalization, regardless of the location of initial admission or healthcare setting, are needed. This may include the expansion of more agile networks for patient transfers when these are not readily available.

Translational outlook. The performance of in-hospital coronary revascularization rates should be prospectively evaluated as a system quality measure for the management of NSTE-ACS. 


\section{REFERENCES}

1. Rossello X, Huo Y, Pocock S, et al. Global geographical variations in ST-segment elevation myocardial infarction management and post-discharge mortality. Int J Cardiol 2017;245:27-34.

2. Roffi M, Patrono C, Collet JP, et al. 2015 ESC Guidelines for the management of acute coronary syndromes in patients presenting without persistent ST-segment elevation: Task Force for the management of acute coronary syndromes in patients presenting without persistent ST-segment elevation of the European Society of Cardiology (ESC). Eur Heart J 2016;37:267-315.

3. Amsterdam EA, Wenger NK, Brindis RG, et al. 2014 AHA/ACC Guideline for the management of patients with non-ST-elevation acute coronary syndromes: a report of the American College of Cardiology/American Heart Association Task Force on practice guidelines. J Am Coll Cardiol 2014;64:e139-e228.

4. Hall M, Dondo TB, Yan AT, et al. Association of clinical factors and therapeutic strategies with improvements in survival following non-ST-elevation myocardial infarction, 2003-2013. JAMA 2016;316:1073-82.

5. Puymirat E, Battler A, Birkhead J, et al. Euro Heart Survey 2009 snapshot: regional variations in presentation and management of patients with AMI in 47 countries. Eur Heart J Acute Cardiovasc Care 2013;2:359-70.

6. Bueno H, Danchin N, Tafalla M, Bernaud C, Annemans L, Van de Werf F. EPICOR (long-tErm follow-up of antithrombotic management Patterns In acute CORonary syndrome patients) study: rationale, design, and baseline characteristics. Am Heart J 2013;165:8-14. 
7. Huo Y, Lee SW, Sawhney JP, et al. Rationale, design, and baseline characteristics of the EPICOR Asia study (Long-tErm follow-uP of antithrombotic management patterns In Acute CORonary Syndrome patients in Asia). Clin Cardiol 2015;38:511-9.

8. Bueno H, Rossello X, Pocock S, et al. Regional variations in hospital management and post-discharge mortality in patients with non-ST-segment elevation acute coronary syndrome. Clin Res Cardiol 2018;107:836-44.

9. Pocock SJ, Huo Y, Van de Werf F, et al. Predicting two-year mortality from discharge after acute coronary syndrome: an internationally-based risk score. Eur Heart J Acute Cardiovasc Care 2017:2048872617719638 [Epub ahead of print].

10. Schiele F, Gale CP, Bonnefoy E, et al. Quality indicators for acute myocardial infarction: a position paper of the Acute Cardiovascular Care Association. Eur Heart J Acute Cardiovasc Care 2017;6:34-59.

11. Fox KA, Goodman SG, Klein W, et al. Management of acute coronary syndromes. Variations in practice and outcome; findings from the Global Registry of Acute Coronary Events (GRACE). Eur Heart J 2002;23:1177-89.

12. Budaj A, Brieger D, Steg PG, et al. Global patterns of use of antithrombotic and antiplatelet therapies in patients with acute coronary syndromes: insights from the Global Registry of Acute Coronary Events (GRACE). Am Heart J 2003;146:999-1006.

13. Mandelzweig L, Battler A, Boyko V, et al. The second Euro Heart Survey on acute coronary syndromes: characteristics, treatment, and outcome of patients with ACS in Europe and the Mediterranean Basin in 2004. Eur Heart J 2006;27:2285-93. 
14. Bueno H, Pocock S, Medina J, et al. Association between clinical pathways leading to medical management and prognosis in patients with NSTEACS. Rev Esp Cardiol (Engl Ed) $2017 ; 70: 817-24$.

15. Wijeysundera HC, Sidhu MS, Bennell MC, et al. Predictors of initial revascularization versus medical therapy alone in patients with non-ST-segment-elevation acute coronary syndrome undergoing an invasive strategy. Circ Cardiovasc Interv 2016;9:pii: e003592.

16. Chin CT, Ong TK, Krittayaphong R, et al. Characteristics and outcomes of medically managed patients with non-ST-segment elevation acute coronary syndromes: insights from the multinational EPICOR Asia study. Int J Cardiol 2017;243:15-20.

17. Arora S, Matsushita K, Qamar A, Stacey RB, Caughey MC. Early versus late percutaneous revascularization in patients hospitalized with non ST-segment elevation myocardial infarction: the atherosclerosis risk in communities surveillance study. Catheter Cardiovasc Interv 2018;91:253-9.

18. Bainey KR, Kaul P, Armstrong PW, et al. Hospital variation in treatment and outcomes in acute coronary syndromes: insights from the Alberta Contemporary Acute Coronary Syndrome Patients Invasive Treatment Strategies (COAPT) study. Int J Cardiol 2017;241:70-5.

19. Yang Q, Wang Y, Liu J, et al. Invasive management strategies and antithrombotic treatments in patients with non-ST-segment elevation acute coronary syndrome in China: findings from the improving CCC project (Care for Cardiovascular Disease in China). Circ Cardiovasc Interv 2017;10:pii: e004750.

20. Heras M, Bueno H, Bardaji A, Fernandez-Ortiz A, Marti H, Marrugat J. Magnitude and consequences of undertreatment of high-risk patients with non-ST segment elevation 
acute coronary syndromes: insights from the DESCARTES Registry. Heart 2006;92:1571-6.

21. Chung SC, Sundstrom J, Gale CP, et al. Comparison of hospital variation in acute myocardial infarction care and outcome between Sweden and United Kingdom: population based cohort study using nationwide clinical registries. BMJ 2015;351:h3913.

22. Bebb O, Hall M, Fox KAA, et al. Performance of hospitals according to the ESC ACCA quality indicators and 30-day mortality for acute myocardial infarction: national cohort study using the United Kingdom Myocardial Ischaemia National Audit Project (MINAP) register. Eur Heart J 2017;38:974-82. 


\section{FIGURE LEGENDS}

Figure 1. Incidence rate ratios (IRR) in the univariate and multivariate Poisson regression models for the $\mathbf{1 5}$ predictors of 2-year all-cause mortality for which coronary revascularization rates were adjusted for. Poisson regression models were performed at individual-patient level to obtain each subject's adjusted 2-year mortality rate. After obtaining the estimates, data was collapsed to obtain the mean adjusted 2-year mortality rate for each level of analysis (hospital deciles, countries and world regions) and make correlations between the average revascularization rate for each group in each level of analysis and 2-year adjusted mortality rate.

Central Illustration: Correlation between in-hospital coronary revascularization rate (percutaneous coronary intervention and/or coronary artery bypass grafting) and 2-year, risk-adjusted, all-cause mortality rate, according to hospital decile, country and region. The figures are weighed-data scatter plots illustrating the relationship between 2-year risk-adjusted mortality rates (adjusted for 15 known predictive factors (9)) and coronary revascularization rates by either predefined region (panel A), country (panel B), and hospital decile (panel C). Each circle represents a population group (region, country or hospital decile) and its size is scaled according to its size. The regression lines demonstrate a strong inverse association across the 3 comparisons. 
TABLE 1: Baseline patient characteristics, in-hospital treatment and mortality by hospital level according to coronary revascularization rate (in deciles 1,5 and 10)*

\begin{tabular}{lccc}
\hline & $\begin{array}{c}\text { Hospitals } \\
\text { in Decile 1 }\end{array}$ & $\begin{array}{c}\text { Hospitals } \\
\text { in Decile 5 }\end{array}$ & $\begin{array}{c}\text { Hospitals } \\
\text { in Decile 10 }\end{array}$ \\
$\begin{array}{l}\text { Number of patients in each decile } \\
\text { (\%) }\end{array}$ & $845(7.1)$ & $1,289(10.8)$ & $1,477(12.4)$ \\
\hline Baseline characteristics & & & \\
Age (years), mean (SD) & $62.9(12.0)$ & $62.7(11.3)$ & $62.4(11.5)$ \\
Male, n (\%) & $563(66.6)$ & $907(70.4)$ & $1,128(76.4)$ \\
Hypertension, n (\%) & $585(70.1)$ & $798(62.6)$ & $874(59.5)$ \\
Hypercholesterolemia, n (\%) & $324(41.5)$ & $396(33.1)$ & $461(32.0)$ \\
Diabetes mellitus, n (\%) & $289(34.7)$ & $309(24.3)$ & $363(24.8)$ \\
Currently smoking, n (\%) & $350(45.2)$ & $546(45.7)$ & $564(40.2)$ \\
Prior CVD, n (\%) & $373(47.1)$ & $545(44.0)$ & $514(35.0)$ \\
Clinical status on admission, n (\%) & & & \\
LVEF <40\% & $64(8.7)$ & $59(5.1)$ & $80(5.6)$ \\
Creatinine $\geq \mathbf{1 . 2} \mathbf{~ m g / d l ~}$ & $269(35.5)$ & $305(24.5)$ & $335(23.4)$ \\
Glucose >160 g/dl & $211(28.4)$ & $232(20.5)$ & $255(18.6)$ \\
Hemoglobin <13 mg/dl & $263(35.4)$ & $381(30.7)$ & $388(26.8)$ \\
Patient management, $\mathbf{( \% )}$ & & & \\
Coronary revascularization & $0(0)$ & $724(56.8)$ & $1,428(96.8)$ \\
$\quad$ PCI & $0(0)$ & $694(54.3)$ & $1,411(95.6)$ \\
CABG & $0(0)$ & $34(2.7)$ & $18(1.2)$ \\
DAPT at discharge & $650(76.9)$ & $1,025(79.5)$ & $1,393(94.3)$ \\
Statin at discharge & $712(85.2)$ & $1,150(89.8)$ & $1,382(93.8)$ \\
BB at discharge & $632(75.5)$ & $958(74.5)$ & $1,143(77.4)$ \\
ACEI/ARB at discharge & $601(71.7)$ & $794(61.9)$ & $964(65.3)$ \\
MRA at discharge & $67(8.0)$ & $78(6.1)$ & $109(7.4)$ \\
2-year mortality & $78(9.2)$ & $79(6.1)$ & $53(3.6)$ \\
\hline
\end{tabular}

*For a simpler presentation, this table only shows deciles 1,5 and 10 . Data for all 10 deciles are shown in Supplementary Table 1.

ACEI/ARB angiotensin-converting enzyme inhibitor/angiotensin receptor blocker; BB, betablocker; $\mathrm{CABG}$, coronary artery bypass grafting; CVD, cardiovascular disease; DAPT, dual antiplatelet therapy; LVEF, left ventricular ejection fraction; MRA, mineralocorticoid receptor antagonist; PCI, percutaneous coronary intervention; SD, standard deviation. 